\title{
TRANSFORMACIONES SOCIALES EN EL NEOLITICO FINAL DE LA EUROPA TEMPLADA (4000-2000 a.C.) (1)
}

POR

\author{
KRISTIAN KRISTIANSEN (*)
}

RESUMEN Se examinan los cambios en el registro arqueológico de la Europa templada desde los complejos culturales TRB/Megalítico al de la Cerámica cordada/Hacha de Combate/Tumba individual atendiendo a las transformaciones en los patrones de subsistencia (agricultura de rozas vs. pastoreo), distribución del excedente (festejos (2) vs. intercambio de riqueza) y prácticas rituales (colectivas vs. individualizadoras).

ABSTRACT Changes in the archaeological record of temperate Europe from the TRB/Megalithic to the Corded/Ware/Battle/Axe/Single Grave cultural complexes are examined in terms of transformations in subsistence patterns (slash-and-burn farming vs. pastoralism), surplus distribution (feasting vs. wealth exchange), and ritual practices (collective vs. individualizing).

Palabras clave Neolítico, Europa septentrional, megalitos, cerámica cordada, hachas, pastoreo, agricultura de roza, excedente, rituales colectivos e individualizadores.

\section{INTRODUCCION}

Si adoptamos un punto de vista general, el Neolítico europeo se caracteriza por la sucesión de tres secuencias culturales principales: la Cultura de la Cerámica de Bandas en el quinto milenio, las Culturas Megalítica y TRB de Europa occidental y septentrional en el cuarto milenio y las Culturas de la Cerámica Cordada y Campaniforme en el tercer milenio (Champion et al. 1984, caps. 5-6). La

(') Center for Research in the Humanities. Copenhagen University.

(1) Este artículo se presentó originariamente a la reunión del cincuenta aniversario de la Society of American Archaeology, Denver, mayo 1985, en una sesión organizada por John C. Cross \& Dean J. Saitta titulada: La organización de la producción en sociedades no estratificadas. Mis ideas en gran medida recogen los resultados de las discusiones y publicaciones durante la última década de Niels Andersen, David Braun, Richard Bradley, Antonio Gilman, Torsten Madsen, Poul Otto Nielsen, Steve Shennan, Andrew Sherrat, Jørgen Skaarup, Nick Thorpe y Chris Tilley. Quiero agradecer a Antonio Gilman, Poul Otto Nielsen y Christopher Tilley sus útiles comentarios al manuscrito de Denver.

La traducción para esta revista ha corrido a cargo de los Dres. A. Gilman Guillén (California State University. EE.UU.), T. Chapa Brunet (Universidad Complutense) y M. ${ }^{a}$ I. Martínez Navarrete (CSIC). El texto ha sido supervisado por el autor.

(2) N. del T. Los términos "feasting» y ufeast" han sido traducidos en todos los casos como "fiestas" o «festejos». En inglés dichos términos implican el consumo de comida en un contexto ritualizado. 
Cultura de la Cerámica de Bandas, de finales del sexto y quinto milenio, refleja la expansión inicial de una agricultura que ocupaba las áreas centrales fértiles, siguiendo el curso de los ríos y las productivas tierras loésicas de Europa central. Unos diez siglos después de esas secuencias culturales ininterrumpidas, en el cuarto milenio, se produce una importante transformación y expansión de las comunidades neoliticas en la mayor parte de Europa septentrional y occidental, caracterizada por la construcción de monumentos megaliticos. Refleja la saturación del paisaje en la Europa templada. En Europa central esta fase también se define por el primer uso de útiles de cobre, mientras que los megalitos siguen siendo un rasgo fundamental de la Europa occidental y septentrional. Durante todo el cuarto milenio se desarrolló un cierto número de culturas neoliticas regionales y más tarde locales. A comienzos del tercer milenio, les sucedió una nueva oleada cultural importante que afecta a la mayor parte de la Europa templada con cerámica cordada, hachas de combate y tumbas individuales cubiertas por pequeños túmulos. La expansión de la Cultura de la Cerámica Cordada (en Escandinavia se llama Cultura del Hacha de Combate/Tumba Individual), seguida por las de los campaniformes y la primera tecnología metalúrgica, refleja asimismo la intrusión de un régimen de subsistencia básicamente pastoril en las áreas más marginales de la Europa templada.

A continuación vamos a ocuparnos primordialmente de la segunda y tercera secuencias, las Culturas Megalíticas/TRB y las Culturas Campaniformes/Tumba Individual/Hacha de Combate y de su transformación. Ambas culturas han sido denominadas a partir de sus rasgos rituales más destacados: monumentos funerarios y cerámica. Tradicionalmente las Culturas Megalíticas fueron concebidas como el resultado de un proceso de difusión desde el Mediterráneo occidental, mientras la Cultura de la Tumba Individual se interpretaba como reflejo de una importante migración de pueblos de lengua Indo-europea desde las estepas rusas. Durante los últimos veinte años, sin embargo, algunos estudios regionales y locales han hecho posible reconstruir los procesos internos relativos a las formas de ocupación del territorio, la economía y la ecología. De acuerdo con ello, podemos pensar que la secuencia que arranca de los megalitos y llega a las tumbas individuales refleja un proceso de intensificación económica y de expansión de la ocupación. En particular Colin Renfrew (1973) ha demostrado las funciones internas de los megalitos como indicadores territoriales de un paisaje agrícola cada vez más poblado con una creciente competencia por la tierra. A su vez, Andrew Sherratt (1981) ha puesto de manifiesto cómo ciertas innovaciones tecnológicas importantes, que denominó "revolución de los productos secundarios", tuvieron lugar durante los procesos de expansión que definieron la transformación del cuarto al tercer milenio en la Europa templada (véase una revisión crítica en Chapman 1982). Esta revolución incluyó el empleo de animales de tiro para uncir el arado y a las carretas, así como el aprovechamiento de productos lácteos y la manufactura textil y lanar. Este proceso marca igualmente un cambio geográfico por el cual se pasa de los buenos suelos explotados más intensivamente de Europa septentrional y occidental, caracterizados por la arquitectura megalítica, a las áreas más marginales y menos fértiles, cuya explotación estaba basada primordialmente en la cría de ganado (véase también Sherratt, 1984).

En consecuencia, conocemos hoy bastante bien las secuencias culturales, económicas y ecológicas del cuarto al tercer milenios de la Europa templada (3), aunque todavía no podemos explicar la rápida transformación y la coincidente y amplia expansión de nuevos rasgos culturales en las fases iniciales tanto de los megalitos como de las tumbas individuales.

Asimismo se han publicado recientemente nuevas interpretaciones acerca de las propiedades internas de la organización social. Se ha propuesto que estamos ante dos variantes de una estructura tribal, cuyas propiedades sociales e ideológicas internas se vinculaban con diferencias económicas y ecológicas (Kristiansen, 1982). En los paisajes boscosos del cuarto milenio la tierra agrícola era un recurso escaso, cuya creación y mantenimiento requería un esfuerzo colectivo coordinado. La construcción de los monumentos megalíticos se basaba en el mismo tipo de organización colectiva

(3) En el caso de Europa puede encontrarse un resumen más justificado de la evidencia en Whittle (1985) y Barker (1985) y, en el de Dinamarca, en Hedeager y Kristiansen (1988). El marco ecológico, que es básico para una comprensión de los cambios económicos y sociales, no está resumido en un sólo trabajo. Hay buenas discusiones, desarrolladas por algunos botánicos destacados, en relación con el pólen europeo (Behere, 1986), la palinologia inglesa (Simmons y Tooley 1981) y danesa (Andersen et al, 1985; Andersen, 1988a y 1988b). 
del trabajo que la necesaria para clarear la tierra. En términos ideológicos, dicha construcción reflejaba además la limpieza de piedras de los campos y, en términos rituales, la construcción de casas para el muerto similares a las de los vivos. Todo esto era parte de un elaborado sistema religioso de culto al antepasado, que destacaba la continuidad entre el pasado y el presente, la vida y la muerte (Kristiansen, 1984), una prolongación ritualizada de la estructura de linaje comunal. Se hacia valer lo colectivo más que lo individual (Shanks y Tilley, 1982).

Por el contrario, las tumbas individuales del tercer milenio expresaban un medio ecológico muy distinto. Principalmente dominaban el paisaje amplias extensiones de pastos con manchas de avellanos y robles. La tierra era abundante. La economia era fundamentalmente pastoril, basada en la cria de rebaños de animales que pastaban en libertad y cuya alimentación se completaba con algunos productos agrícolas. La organización social, consecuentemente, no acometía importantes esfuerzos colectivos como el clareado del bosque o la demarcación de su territorio mediante la construcción de monumentos. La unidad básica de producción era la familia que, en términos ideológicos, quedaba reflejada en los pequeños túmulos familiares funerarios con tumbas individuales. El individuo y su propiedad son ahora el foco de los ritos funerarios. Se subraya con fuerza un rango personal que no se disimula en los monumentos y ritos colectivos (Shennan, 1982).

Esas variantes de la estructura tribal propias de la Eurasia templada durante el cuarto y tercer milenios han sido denominadas por Colin Renfrew (1976) jefaturas con un poder basado en lo colectivo y en lo individual, respectivamente. Otros han recalcado la relación entre organización social y ritual - la Cultura Megalítica se designa como "estructura de autoridad ritual» y la Cultura de la Tumba Individual como *economía de bienes de prestigion (Thorpe y Richards, 1984)-. Pero, aunque ello representa importantes intuiciones, no nos acerca a una comprensión ni de su dinámica interna, ni de la articulación de la producción con los excedentes y la jerarquía. Dicha comprensión creo que es una condición previa necesaria si queremos explicar su transformación así como los principales procesos de difusión que tuvieron lugar durante ese período (4)

En un estudio reciente he intentado presentar la articulación de la producción, el excedente y la organización social en la Cultura Megalítica del cuarto milenio y en la Cultura de la Tumba Individual del tercero, respectivamente (Kristiansen, 1984). Antes de explicar el modelo debería quizás clarificar brevemente mi concepción de excedente y consumo.

En sociedades previas a la estratificación, un excedente satisface necesidades básicamente sociales, por lo tanto no podemos separar nuestra definición de excedente de nuestra definición de la organización social de la producción. Considero, pues, que el excedente forma parte consustancial del control social del trabajo y de la producción, y de la subsiguiente distribución de la segunda. Esta distribución puede lograrse mediante los festejos, la manufactura y circulación de productos valiosos o el almacenamiento de un excedente potencial de rebaños o cosechas. Tal excedente puede ser transformado de diversas formas en poder, prestigio o rango. En este proceso de transformación, el consumo juega un papel importante, tanto al crear una dependencia, como al limitar el acceso a esos artículos consumidos, ya sean alimento, ganado o bienes de prestigio. El consumo es, a la vez, un mecanismo de regulación y de manipulación y es el proceso de consumo el que crea los residuos que son la base primordial para la interpretación de los arqueólogos.

En las sociedades tribales, el ritual es una de las formas más potentes y más comunmente empleadas de legitimar la transformación del trabajo y de la producción en poder político y prestigio. Así pues, es crucial la interpretación de las funciones materiales del ritual y de la religión para comprender la articulación de la producción, el excedente y la jerarquización. De ello se deriva también que cualquier discusión de conceptos tales como excedente y consumo implica la consideración de la totalidad de la evidencia arqueológica.

(4) En un interesante estudio reciente, Julian Thomas (1988) intenta explicar la estabilidad y cambio en la Europa neolítica atendiendo a dos tipos básicamente diferentes de organización social -el modo de linaje y el modo germánico-, el último derivado, probablemente, de la organización social Indoeuropea. Como definen las relaciones sociales de un modo diferente en lo que se refiere a la tierra, la propiedad y la producción, implican estrategias sociales y potencial organizativo diferentes.

Este estudio del parentesco y la reproducción social en la Europa prehistórica puede conectarse con las observaciones bastante similares de Goodys (1976) a propósito de la historia más reciente. 
En la Cultura Megalitica, subsistencia, organización social y religión estaban estrechamente ligadas entre si en una estructura vertical ritualizada de reproducción. La estrecha conexión entre ritual, subsistencia e intercambio queda demostrada por el papel jugado por el hacha pulimentada de silex en las tres áreas. Como instrumento básico en la subsistencia, medio fundamental de intercambio y medio principal de depósito ritual, el hacha aúna la producción, el intercambio y el consumo ritual/festivo. Las hachas sirvieron como medio de intercambio social y consumo ritual y fueron, a la vez, una condición previa para un incremento de la producción al emplearse para el clareado de nuevos bosques. Esto podia producir, de nuevo, el excedente suficiente para dar fiestas y para participar en alianzas y crear depósitos rituales, incrementando así el control ritual (= social) y el prestigio.

Así, la ampliación ritualizada de producción y prestigio a través de los festejos y el depósito ritual de hachas pulimentadas revela las funciones materiales principales de esas instituciones culturales. Además nos indica cómo el excedente de producción se introduce en el ciclo local de creación del prestigio. La transformación del excedente de producción en festejos rituales, alianzas y prestigio desarrolló un sistema jerárquico ritualizado que sirvió durante más de medio milenio para organizar con éxito la producción de un sistema de asentamiento disperso de agricultura de roza. Estuvo respaldado por el empleo de megalitos y lugares rituales centrales en un complejo y rígido sistema religioso territorial de culto a los antepasados. Dicho sistema, al definir el rango y la descendencia en relación con los ancestros fundadores, legitimó la desigualdad en el acceso a y el control de la tierra, del trabajo y del excedente de producción. También se ha pensado que la naturaleza limitada del sistema y sus contradicciones potenciales quedarian reflejadas en la estructura del diseño y la decoración cerámicas (Hodder, 1982; Tilley, 1984).

En la subsiguiente Cultura de la Tumba Individual la conexión entre ritual, intercambio y subsistencia marcada por la Cultura Megalítica queda disuelta. El hacha pulimentda de sílex todavía es el instrumento más importante para la producción subsistencial, pero no se emplea en el intercambio social, ni en el consumo ritual. El excedente y la riqueza ya no se almacenan en los productos de la tierra sino, más bien, en rebaños de ganado vacuno y ovejas. También se disuelve la organización territorial basada en los megalitos y los centros rituales. En lugar de ella vemos un patrón de asentamiento disperso de pequeñas aldeas autónomas, cada una de las cuales construye sus propios pequeños túmulos funerarios. La Cultura de la Tumba Individual estimula una enorme producción local de hachas de combate, el equipo normalizado en los enterramientos masculinos. En las fases iniciales, las hachas, como algunos tipos cerámicos, son bastante similares por toda Europa central y septentrional, pero más tarde se ven sometidas a un desarrollo local, que refleja ciclos cada vez más cerrados de intercambio y matrimonios cruzados.

La información que transmiten los artículos de prestigio y el ganado prima así sobre los bienes y útiles básicos, uniendo ámplias áreas de la Europa templada y creando una cultura material bastante similar desde Ucrania a la costa atlántica, cuya composición regional, sin embargo, es variada. Los campaniformes y las hachas fueron importantes manifestaciones ideológicas de estrategias predatorias y socializantes: la guerra y el consumo de cerveza (Burgess y Shennan, 1976). Lo que observamos, entonces, es una organización social adaptada a una expansión predatoria, y la incorporación de nuevos grupos durante todo el proceso (véase Sahlins, 1961). No hay evidencia de una jerarquía fija. Se ha sugerido también que el estilo cerámico refleja una organización social abierta, no delimitada (Hodder, 1982; Tilley, 1984). El equipo de estatus personal de los jefes en las tumbas, coincidente en amplias zonas de la Europa templada, está en concordancia con una estrategia social de ese tipo (Shennan 1986a y 1986b). La competencia, la autonomía y el cambio de alianzas habian reemplazado a la organización inicial rígida y territorialmente estable. La competencia se manifestaba abiertamente en el ritual funerario en lugar de ocultarse y canalizarse como en el ritual colectivo de los megalitos. Debe hacerse notar, sin embargo, que aunque la Cultura de la Tumba Individual sea menos jerárquica que la Cultura Megalítica, estamos tratando todavía con una organización tribal donde el rango y el estatus eran propiedades fundamentales de la organiza- 
ción social, pero podian ser empleados más libremente en una red abierta de alianzas cambiantes $\mathrm{y}$ agregaciones politicas.

Cuando se comparan esas dos variantes de la estructura tribal, vemos que están operando dos principios diferentes (Fig. 1) (Friedman, 1975). En la Cultura Megalitica, el control del trabajo y la conversión de la producción en festejos y creación de prestigio eran una condición previa para tomar parte en las alianzas y en el intercambio. Las relaciones verticales que conectaban los linajes con los antepasados de la propia comunidad que reposaban en los megalitos eran el principio dominante para la definición de toda fiesta e intercambio. En este ciclo, los bienes de prestigio son una variable dependiente. Todas las formas de acumulación están subordinadas a la capacidad de dar fiestas, para demostrar a lo sobrenatural el poder de sus propios linajes. Expresa un control ritualizado del trabajo y la reproducción social.
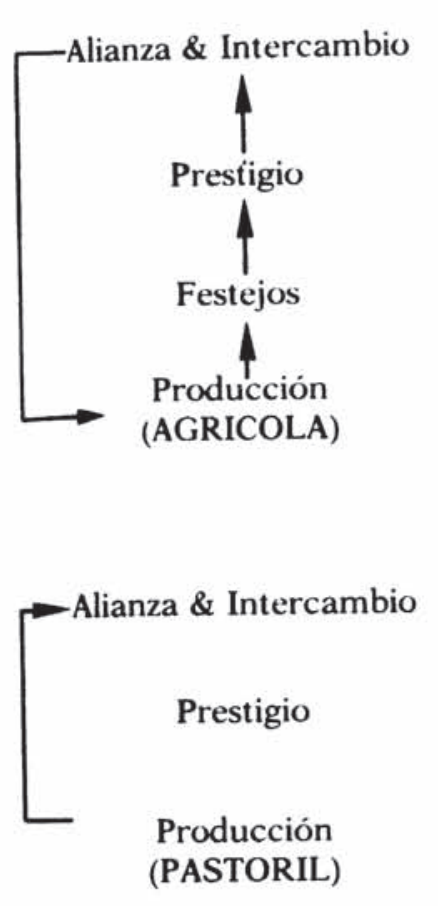

Hachas de silex \& pocos bienes de prestigio

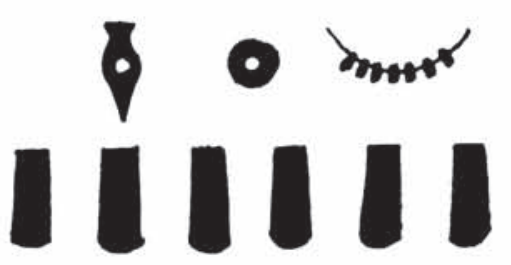

Bienes de prestigio \& pocas hachas de sílex

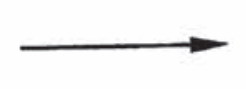


Debe recalcarse, sin embargo, que el funcionamiento de los bienes de prestigio en la organización social de la Cultura de la Tumba Individual no es explicable sin referirlo a las fases previas que transformaron las relaciones entre producción, ritual y organización social como resultado de procesos históricos específicos. Hasta ahora he descrito las dos estructuras como si se opusieran la una a la otra. Intentaré ahora bosquejar las condiciones para su transformación.

La edificación de cientos de megalitos a mediados del cuarto milenio representó la culminación de un proceso de expansión agricola de la población y de las formas de ocupación del espacio, proceso que se estabilizó en un patrón territorial consistente en una jerarquia tribal ritualizada en evolución. La estructura permaneció intacta durante otro medio milenio, pero bajo una presión interna creciente. Puede sugerirse que el equilibrio entre la explotación de la tierra agrícola y del bosque fue alterado gradualmente, ya que el suelo forestal se agotó. El aumento de población fue un factor adicional, pero dependiente de las propiedades de las relaciones de producción. En la Cultura Megalítica esto se reflejaba en la elevada demanda de movilización del excedente y del trabajo para dar fiestas y crear prestigio.

Durante los últimos siglos de la Cultura Megalítica aumentaron los campos abiertos y la cría de animales (Madsen, 1982, fig. 17), una tendencia aparentemente general en Europa (Kruk 1980; Sochacki, 1980: 98 y ss). Los asentamientos se hicieron mayores y más permanentes (Skaarup, 1985) (5). Además la construcción de megalitos y lugares centrales rituales se detuvo y, al mismo tiempo, el ritual y el consumo colectivos decrecieron. En la cerámica ritual esto se manifiesta en una disolución gradual de los patrones decorativos limitados (Hodder, 1982; Tilley 1984). Puede proponerse que este proceso representa el cambio desde un control ritualizado de la reproducción social, en el marco de una forma dispersa de ocupación del territorio, a un control doméstico inserto en un asentamiento centralizado con menor necesidad de ritualizar el poder y la autoridad. La ideología de los constructores de megalitos intentaba ocultar dicho proceso mediante una afiliación territorial continua con la comunidad a través de ritos en los sitios tradicionales, y de la introducción de nuevos enterramientos en los antiguos megalitos. A largo plazo, la contradicción entre un sistema de organización social jerárquico ritualizado y las condiciones reales de producción en un medio crecientemente degradado, en algunas áreas, llevó a una dispersión de los núcleos rurales y de la población, que se expandió rápidamente hacia nuevos territorios. Estos se encontraron en medios menos fértiles de Europa central y septentrional, que podian ser transformados fácilmente en pastos para la cría de animales.

Para comprender la dinámica de unos cambios a tan gran escala hay que tener en cuenta los beneficios económicos de una producción más dependiente de la cría de animales. Dadas las condiciones ecológicas y las limitaciones de la agricultura primitiva sin abonado, en un habitat templado abierto con bosques y suelos que se degradan lentamente (6), la cría de animales que pacen en libertad es, con mucho, la estrategia intensiva más productiva y de menor esfuerzo (Fleming, 1972). La inestabilidad y la expansión son rasgos inherentes a una economía de ese tipo que llegó a predominar en la mayor parte de Europa durante el tercer y segundo milenios, incluso en el Mediterráneo (Harrison y Moreno López, 1985). Los animales son móviles, su reproducción y expansión es más fácil que las de una producción agrícola. Esto lleva o bien a una explotación excesiva de los pastos y/o a una expansión que explica la naturaleza guerrera y el aparente dominio masculino de las sociedades pastoriles, en general, y de las sociedades europeas durante el tercer y

(5) Este desarrollo, combinado con alguna contracción y desplazamiento del asentamiento, puede explicar la tendencia general hacia una recuperación del bosque por toda Europa septentrional, antes de la expansión de los grupos de la Cerámica Cordada/Hacha de Combate. Berglund (1969) y Gorransson (1988) lo discuten en relación con Escandinavia y Bradley (1978: 106 y ss.) con Inglaterra.

(6) No hay evidencia que confirme la propuesta de Rowley-Convy (1980) de que el «landnam» del cuarto milenio de Europa septentrional estuviera basado en campos permanentes, sino más bien al contrario, como confirmó la evidencia botánica reciente (Andersen, 1988b). Los campos abonados de modo permanente corresponden a la tradición más antigua de la Cultura de la Cerámica de Bandas y a algunos de sus descendientes de inicios del cuarto milenio (Troels-Smith, 1984). Sin embargo, es correcta la observación de Rowley-Convy relativa al carácter permanente del clareado del bosque. 
segundo milenios, en particular. Ello se enraiza también en la división del trabajo, en la cual el hombre se ocupa de los rebaños que son ahora el elemento dominante en la economia (7). En la Europa prehistórica, sin embargo, la agricultura sirvió como un factor de equilibrio. Asi, hubo una economía mixta de cría de ganado y agricultura en un paisaje abierto de pastos y bosque secundario mixto.

En el nuevo medio podia tener lugar una competencia sin restricciones. Pero, como el control ritual de la tierra y de la producción local ya no era crucial, se desarrollaron nuevos medios de conseguir estatus que estuvieran adaptados a las nuevas condiciones sociales y económicas de producción, caracterizadas por unidades de asentamiento autosuficientes y una riqueza móvil. Como consecuencia de ello, dominó la ideologia que justificaba la exhibición del estatus y prestigio individuales. Sin embargo, como condición histórica previa, la Cultura TRB tenía que haber establecido el estatus y el rango como propiedades fundamentales del paisaje social neolítico.

Dado que la nueva estructura estaba basada en la competición y la expansión, tendia a socavar y dominar "la estructura de autoridad ritual» territorial más estática de la Cultura Megalítica. Los dos sistemas fueron así mutuamente contradictorios o incompatibles. Esto tuvo importantes implicaciones en la forma en que se extendió el Complejo Cultural de la Cerámica Cordada. Su primera fase se estableció rápidamente en las periferias de las áreas tradicionales de asentamiento de la Europa templada (Buchvaldek, 1980; Kruk, 1980; Sherratt, 1981; Starling, 1985). Sólo en esas áreas encontramos todos los rasgos diagnósticos. Después, gradualmente, penetraron en las antiguas áreas nucleares de las más antiguas culturas neolíticas, pero adoptando sólo parcialmente la estructura ritual existente. En todo caso, fue la segunda oleada - campaniformes y puñales- la que completó el proceso de integración cultural (Harrison, 1980).

Así, el proceso de transformación dependió de una integración entre el intercambio local y el regional, entre las fuerzas internas y las externas, que todavía no se ha comprendido adecuadamente (véase Kristiansen, 1988). En Escandinavia, pueden distinguirse tres variantes:

1. Areas con un asentamiento previo escaso - a menudo marginal - caracterizadas por un Complejo Cultural de Cerámica Cordada/Tumba Individual/Hacha de Combate, por ejemplo, Jutlandia/Schleswig-Holstein (Glob, 1944, Struve, 1955).

2. Areas con una ocupación de la Cultura TRB que adopta partes importantes del Complejo Cultural de la Tumba Individual, que lleva la formación de culturas nuevas y originales, por ejemplo, Suecia (Malmer, 1962) (8)

3. Areas con ocupación TRB que rechazaron el Complejo Cultural de la Tumba Individual y sólo muy lentamente adoptaron algunos aspectos, pero básicamente permanecieron intactas, excepto en muy pequeñas bolsas de expansión, hasta la fase Campaniforme/Puñal, por ejemplo las islas danesas/Mecklemburg (Ebbesen, 1982-83; Andersen Hedegaard, 1986; Nilius, 1981).

Aunque la Cultura Megalítica más tardía había experimentado un aumento demográfico, cierta degradación ecológica y el desarrollo de contradicciones entre las bases económicas y la organización social ritualizada de la producción, la rápida formación y expansión de la Cultura de la Cerámica Cordada no pudo haber ocurrido solamente como resultado de una serie de desarrollos independientes. Era necesario un factor unificador para provocar una expansión del poblamiento tan importante, y la subsiguiente extensión de un complejo social y cultural nuevo y extremadamente homogéneo. El factor desencadenante estaba localizado en esas áreas de Eurasia donde la transfor-

(7) Debe señalarse que las mujeres fueron enterradas en términos iguales a los hombres, en el túmulo familiar, con los niños en la periferia del túmulo. La monogamia es dominante, como se documentó en muchos túmulos familiares (Glog, 1944). La riqueza de las tumbas femeninas, sin embargo, parece depender de la del hombre ya que como esposas fueron enterradas, aparentemente en muchos casos, junto con sus maridos.

(8) En el caso de Suecia, las evidencias recientes sugieren que hubo un influjo de jefes foráneos, probablemente en búsqueda de metal (Janzon, 1986). 
mación del paisaje y de la organización social habian precedido a las de Europa septentrional y occidental, donde se habia desarrollado desde hacia tiempo una economia más pastoril (Anthony, 1986; Kristiansen 1988) (9).

Los grupos tribales que emigraban a través de los pasillos de bosque abierto, y siguiendo el curso de los valles fluviales de la Europa templada, introdujeron una nueva práctica tecnológica, económica y ritual. Como había condiciones internas preparadas para un cambio en amplias áreas de Europa central y septentrional, la nueva economía e ideologia se extendió rápidamente, liberando las contradicciones de las culturas neolíticas. Esto probablemente provocó, de nuevo, ciertas migraciones locales y regionales. En los siglos subsiguientes, el resto de Europa fue afectada por este proceso, creándose de ese modo una base social homogénea para la expansión posterior de la tecnología del bronce y el desarrollo de élites tribales (Gilman, 1981; Kristiansen, 1987; Rowlands 1985; Shennan, 1986a y 1986b). El proceso puede haber sido fomentado también por una oscilación climática (Gräslund, 1980).

Así, en el Neolítico final europeo, la estratificación no fue provocada por una inversión agrícola creciente en la tierra, como Gilman (1981) propuso para Europa meridional, sino más bien por el potencial de control económico y social implícito en una economía de prestigio, de riqueza transportable y de guerra móvil. La inversión en la tierra ès menor en una economía dominada por la cría de animales que en otra en que impera la agricultura. En consecuencia, una economía pastoril, a largo plazo, tiende a someter y transformar las sociedades agrícolas hasta que alcanza sus limites ecológicos y requiere una reinversión en la tierra, como ocurrió durante la Edad del Bronce Final en Europa central y en la transición a la Edad del Hierro en Europa septentrional (10).

La trayectoria evolutiva de la Europa prehistórica del Neolítico y la Edad del Bronce comienza con unas comunidades agrícolas estables con elevadas inversiones en la tierra, producción agrícola importante y jerarquias territoriales en desarrollo, que son gradualmente reemplazadas por sociedades más descentralizadas y pastoriles. Esos procesos fueron un resultado, a largo plazo, de las modificaciones de las condiciones ecológicas de producción y de una cierta interacción con sociedades pastoriles ya establecidas de Eurasia (Anthony, 1986; Gimbutas, 1979) que, a su vez, estaban interactuando con centros de civilización del Cáucaso y Anatolia.

Vistas desde esta perspectiva, podemos considerar las migraciones y la amplia difusión de nuevos elementos culturales como un rasgo estructural de importantes transformaciones sociales. Tal perspectiva implica, además, que tenemos que valorar los diversos desarrollos europeos como parte de oscilaciones evolutivas a gran escala y de interacciones entre Europa, Asia y el Próximo Oriente, cuya naturaleza no se comprende todavia suficientemente (Rowlands, Larsen y Kristiansen, 1987).

(9) Cierto número de investigadores, ingleses sobre todo, han recalcado la importancia de los factores internos en la ruptura y transformación de las comunidades neolíticas de Europa oriental (Sherratt, 1982; Tringham, 1978; Nandris, 1978. Véase también una buena discusión en Bintliff, 1983, notas 57 y 58).

(10) Esto planteá la cuestión del estatus evolutivo y la significación de una agricultura de base pastoril y tribal. Puede apuntarse que representa un estadio general de evolución tribal en habitats de degradación ecológica, con escasa o ninguna posibilidad de un control centralizado de recursos, una trayectoria evolutiva diferente a la de los centros agrícolamente fértiles de civilización (se discute en Engels, 1884/197; Kristiansen, en prensa). 


\section{BIBLIOGRAFIA}

Andersen, S. T.; Aaby, B. and OdgaARD, B. Vad (1983): «Environment and Man. Current Studies in Vegetational History at the Geological Survey of Denmarkw. Journal of Danish Archaeology, 2: 184-196.

ANDERSEN HedegaARD, A. (1986): «Enkeltgravstid på de danske đen». En C. Adamsen y K. Ebbesen, (eds.): Stridss $\emptyset$ ksetid i Sydskandinavien, 57-77. København.

ANDERSEN, S. T. (1988)a: «Natural and cultural landscapes since the Ice Age shown by pollen analyses from small hollows in a forested area in Denmark». To appear in Journal of Danish Archaeology, 8.

- (1986)b: «Evidence of slash and burn cultivation in the Neolithic of Denmark. Pollen spectra from a double passage grave Klekkendehøj on Møn*. To appear in Journal of Danish Archaeology, 7.

Anthony, D. W. (1986): "The 'Kurgan' Culture, Indo-European Origins and the Domesticaton of the Horse: A Reconsiderationw. Current Anthropology, 27, 2: 291-313.

BARKer, G. (1985): Prehistoric Farming in Europe. Cambridge University Press.

BEHRE, K.-E. (ed.) (1986): Anthropogenic Indicators in Pollen Diagrams. Rotterdam/Boston.

BERGLUND, B. (1969): "Vegetation and human influence in South Scandinavia during prehistoric time». Oikos Suppl. 12: 9-28.

BintuIfF, J. (1983): «The Neolithic of Europe and social evolution». En J. Bintliff (ed.). European Social Evolution. Archaeological Perspectives. University of Bradford. Bradford.

Bradley, R. (1978): The Prehistoric Settlement of Britain. Routledge \& Kegan, London.

BuchVAldeK, M. (1980): «Corded Pottery Complex in Central Europe». Journal of Indoeuropean Studies, 8, 3-4: 393-406.

Burgess, C. y SHENNAN, S. (1976): «The Beaker phenomenon: some suggestions». En C. Burgess y R. Miket (eds.): Settlement and Economy in the third and second millennium B. C. BAR 33: 309-326. Oxford.

Chapman, J. C. (1982): "The 'Secondary Products Revolution' and the limitations of the Neolithic». Bulletin of the Institute of Archaeology, University of London, 19: 107-122.

Champion, T., Gamble, C., Shennan, S. y Whittle, A. (1984): Prehistoric Europe. Academic Press. New York.

Engels, F. (1891/1977): Familiens, privatejendommens og statens oprindelse. Politisk Revy, oversat efter 4. udgaven (Danish translation of first edition from 1891 of «The Origin of Family, Private Property and the State», with a new enlarged preface by Engels).

Friedman, J. (1975): «Religion as Economy and Economy as Religion». Ethnos 1-4. Uppsala. Reprinted en J. Friedman (1979): System, Structure and Contradiction. The Evolution of 'Asiatic' Social Formations. The National Museum of Denmark. Copenhagen.

Gilman, A. (1981): "The development of social stratification in Bronze Age Europe». Current Anthropology, 22, 1: $1-23$.

GimbutaS, M. (1979): "The three waves of the Kurgan people in Old Europe, 4500-2500 B. C.». Archives suisses d'anthropologie générale, Géneve, 43, 2: 113-137.

GLoB, P. V. (1944): Studier over den jyske Enkeltgravskultur. (french summary: études sur la civilisation des sepultures individuelles de Jutland). Aarbøger for Nordisk Oldkyndighed og Historie K $\phi$ ben.

Goody, J. (1976): Production and Reproduction. A comparative Study in the Domestic Domain. Cambridge University Press. Cambridge.

Göransson, H. (1988): Neolithic Man and The Forest Environment around Alvastra Pile Dwelling. Theses and Papers in North-European Archaeology, 20. Lund University Press.

GRÄSLUND, B. (1980): "Climatic fluctuations in the Early Subboreal Period. A preliminary discussion". Striae, 14: 13-22.

HARrISON, R. J. (1980): The Beaker Folk. Copper Age Archaeology in Western Europe. Thames \& Hudson. London.

HARRISON, R. J. y MORENO. LÓPEZ, G. (1985): «El policultivo ganadero o la revolución de los productos secundarios". Trabajos de Prehistoria, 42: 51-82. Madrid.

HäUSLER, A. (1983): «Der Ursprung der Schnurkeramik nach Aussage der Grab-und Bestatungssitten». Jahresschrift f. mitteldeutsche Vorgeschichte. 66: 9-30. Halle.

HedeAger, L. y KRISTIANSEN, K. (1988): In Det danske landbrugs historie. Bind L Oldtiden. Landbohistorisk Selskab. København.

HODDER, I. (1982): «Sequences of structural change in the Dutch Neolithic». En Hodder, I. (ed.): Symbolic and Structural Archaeology. Cambridge University Press: 162-177.

JANZON, G. O. (1986): "Stridsyxekulyut och metallurgisk know-hown. En C. Adamsen y K. Ebbesen (eds.): Stridsøksetid $i$ Sydskandinavien: 126-138. Copenhaguen.

Kristiansen, K. (1982): "The Formation of Tribal System in Later Europe 4.000-5.00 B. C.». En C. Renfrew, M. Rowlands y B. A. Seagraves (eds.): Theory and Explanation in Archaeology. New York, Academic Press: 241280.

- (1984): «Ideology and material culture. An archaeological perspectiven. En M. Spriggs (ed.): Marxist Perspectives in Archaeology. Cambridge Univ. Press: 72-100. 
- (1987): „From stone to bronze-the evolution of social complexity in Northern Europe, 2300-1200 BCw. En E. M. Brumfiel y T. K. Earle (eds.). Specialization, Exchange and Complex Societies. Cambridge University Press: $30-51$.

- (1988): "Prehistoric migrations and the case of the Single Grave Culture in Jutland». To appear in: Journal of Danish Archaeology, 8.

- (1988): (in press) «Chiefdoms, states and systems of social evolution». En T. Earle (ed.). To appear in: Chiefdoms. Cambridge University Press and American School of Research.

KRUK, J. (1980): The Neolithic Settlement of Southern Poland. BAR S93. Oxford.

MALmer M. P. (1962): Jungneolitische Studien. Acta Arch. Lundensia Ser. in 8, 2 Lund.

NANDRIS, J. (1978): «Some features of Neolithic climax Socities». Studia Praehorica 1-2, Sofia.

NiLıus, J. (1981): Beitrag zur Stellung der Einzelgrabkultur in Mecklenburg. Jahresschrift mitteldeutsche Vorgeschichte 64: 63-87. Halle.

RENFREW, C. (1973): «Monuments, mobilization and social organization in Neolithic Wessex». En C. Renfrew (ed.) The Explanation of Cultural Change. London, Duckworth: 539-558.

- (1974): «Beyond a Subsistance Economy: The Evolution of Social Organisation in Prehistoric Europen. En Charlotte Moore (ed.) Reconstruction Complex Societies. An Archaeological Colloquium. Supplement to the Bulletin of the American School of Oriental Research, 20:69-98.

RowlandS, M. (1984): "Conceptualizing the European Bronze Age and Early Iron Ages". En J. Bintliff (ed.) European Social Evolution. Archaeological Perspectives. University of Bradford, Bradford: 147-156.

Rowley-ConwY, P. (1981): «Slash and Burn in the Temperate European Neolithicn. En R. Mercer (ed.): Farming Practice in British Prehistory. Edinburgh University Press: 85-96.

Rowlands M., LarSen, M. T., KRISTIANSEN, K. (eds.) (1987): Centre and periphery in the ancient world. Cambridge University Press.

SaHLinS, M. (1961): "The segmentary lineage: an organization of predatory expansion». American Anthropologist, 63: $322-345$.

Shanks, M. T. y Tilley, C. (1982): «Ideology, symbolic power and ritual communication: a reinterpretation of Neolithic mortuary practices». En I. Hodder (ed.): Symbolic and Structural Archaeology. Cambridge University Press: 129-155.

ShennanS, S. (1982): «Ideology, change and the European Early Bronze Agew. En I. Hodder (ed.): Symbolic and Structural Archaeology. Cambridge University Press: 155-161.

- (1986)a: "Central Europe in the third millennium B. C.: an evolutionary trajectory for the beginning of the European Early Bronze Agew. Journal of Anthr. Arch. 5: 115-146. Academic Press.

- (1986)b: «Interaction and change in the third millenium B. C. in western and central Eutropen. En C. Renfrew y J. Cherry (eds.): Peer-polity interaction and sociopolitical change. Cambridge University Press: 137-148.

SHERRATT, A. G. (1981): «Plough and pastoralism: aspects of the secondary products revolution». En I. Hodder et al (eds.): Patterns of the Past. Cambridge University Press: 261-305.

- (1982): «Mobile resources: settlement and exchange in early agricultural Europen. En C. Renfrew y S. Shennan (eds.): Ranking, Resource and Exchange. Cambridge University Press: 13-26.

- (1984): «Social evolution: Europe in the later Neolithic and Copper Ages». In J. Bintliff (ed.): European Social Evolution. Archaeological Perspectives. University of Bradford. Bradford, 123-139.

Simmons, I. y Tooley, M. (eds.) The Environment in British Prehistory. Duckworth. London.

SKAARUP, J. (1985): Yngre stenalder på фerne syd for Fyn. Meddelser fra Langelans Musem. Rudkøbing.

SOCHACKI, Z. (1980): «Some remarks on the social structure and economic system of the Baden Culturen. Journal of Indoeuropean Studies, 8, 3-4: 93-107.

Starling, N . J. (1985): «Social Change in the Later Neolithic of Central Europew. Antiquity, 59: 30-38.

STRUVE, K. W. (1955): Die Einzelgrabkultur in Schleswig-Holstein und ihre kontinentalen Beziehungen. Karl Wachhottz. Neumünster.

TномAS, J. (1988): «Relations of Production and Social Change in the Neolithic of North+West Europe» MAN, vol.

THORPE, I. J. y RICHARDS, C. C. (1984): *The decline of ritual authority and the introduction of Beakers in Britain" En. R. Bradley y J. Gardiner (eds.): Neolithic Studies. BAR 133. Oxford: 67-87.

Tilley, C. (1981-82): An Assessment of the Scanian Battle-Axe Tradition: towards a Social Perspective. Scripta Minora Lund. Gleerup, 1: 5-72.

- (1984): «Ideology and the legitimation of power in the Middle Neolithic of Southern Sweden». En D. Miller y C. Tilley (eds.): Ideology, Power and Prehistory. Cambridge University Press: 111-146.

TRINGHAM, R. (1978): «A re-appraisal of Gordon Childes concepts of the Agricultural and Urban Revolution: recent research in Europe and the USSR». En Shinkin et al (eds.): Anthropology for the Future. Univ. of Illinois.

Troels-Smrth, J. (1984): «Stall-feeding and field manuring in Switzerland about 6.000 years agow. Tools and Tillage v, 1: 13-25.

Whrrtle, A. W. R.(1985): Neolithic Europe: a survey. Cambridge University Press. 